\title{
Anaplastic Childhood Meningioma in a 16-Year-Old Boy
}

\author{
Emel Ebru Pala ${ }^{1}$, Ulku Kucuk ${ }^{1}$, Ebru Arabaci Cakir ${ }^{1}$, Ozcan Binatli ${ }^{2}$
}

\begin{abstract}
Introduction: Meningiomas account for $0.4-4.6 \%$ of all primary brain tumors in children. Childhood meningiomas have several different characteristics than those occuring in adults.

Case: Here we report a 16 year old male presented with long-standing headache and incipient weakness on the left side. Magnetic resonance images revealed a $65 \times 47 \times 37 \mathrm{~mm}$ sized, right sided frontoparietal parasagittal extraaxial mass. Histopathologic examination showed monotonous, small cells with oval/round nucleus forming whorl like structures. Tumor showed high mitotic index and Ki67 proliferation rate around $30 \%$. Immunohistochemically tumor cells were positive with EMA, vimentin, bcl-2, progesterone receptor. The final diagnosis according to histopathological, radiological and immunohistochemical findings was anaplastic meningioma.

Conclusion: High-grade meningiomas are rare in children. Appropriate grading is important, as high grade tumors with high proliferation index are associated with less favorable clinical outcomes and warrant more radical treatment.
\end{abstract}

Keywords: Meningioma, Childhood, High grade

\section{Introduction}

Meningiomas are central nervous system tumors arising from the arachnoid cap cells of the meninges. In adults, meningioma accounts for approximately $30 \%$ of primary CNS neoplasms although child and adolescent meningioma is rare, accounting for $0 \cdot 4-4 \cdot 6 \%$ of CNS tumors [1].

Although the etiological factors of childhood meningiomas were not clarified, a few risk factors have been described, such as Neurofibromatosis -2 (NF2) and radiotherapy [2-3]. Approximately 10.3$40 \%$ of childhood meningiomas are associated with NF2 while it is only $0.35 \%$ for adult meningiomas [4-5]. Gorlin syndrome, Castleman's disease and Rubenstein-Taybi syndrome are the other reported conditions related with meningioma [1].

The mean age at presentation of childhood meningiomas is 10-14.7 years old [6-8]. The median age is younger (10.5 years) for sporadic tumors than those with NF2 (12 years) or radiationinduced meningioma (16 years) [1]. Patients with neurofibromatosis type 2 (NF2) had worse recurrence free survival than those without neurofibromatosis [9]. According to a metaanalysis of case studies, most common localizations of child and adolescent meningiomas are; cerebral convexity, intraventricular, falx and parasagittal regions. Patients present with symptoms due to raised intracranial pressure. Headache,vomiting, papilloedema, monoparesis and seizures are most common ones. Childhood meningiomas have several different features than those occurring in adults such as higher frequency of secondary degeneration (cystic or sarcomatous), male predominance (Male : Female=1.16:1), absence of dural attachment (dural tail sign), higher incidence in the intraventricular location, more agressive behaviour [10]. According to a meta-analysis of 30 studies consisting child/adolescent meningioma cases reports approximately $80 \%$ of the cases are grade1, $11 \%$ grade 2 and $9 \%$ are grade3 [9]. Appropriate grading is important, as high grade tumors with high proliferation index are associated with less favorable clinical outcomes and warrant more radical treatment.

Case: A 16-year-old male was admitted to our hospital with a long standing headache and incipient weakness on the left side. On physical examination suspect left sided hemiparesis was noted. Cranial nerve, sensorial and cerebellar examinations were within normal limits. 


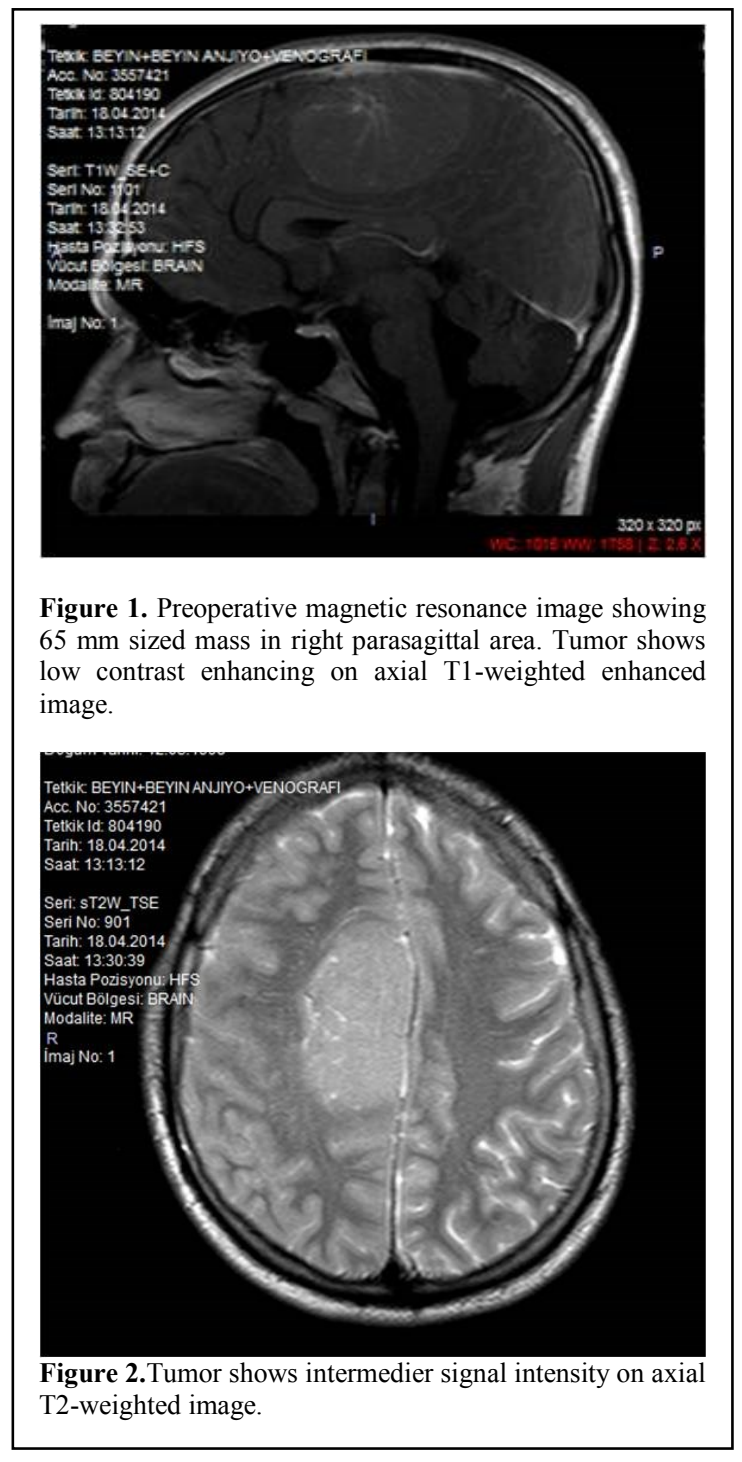

He had no past medical, drug or operation history also stigmata of neurofibromatosis. Magnetic resonance images (MRI) revealed a $65 \times 47 \times 37 \mathrm{~mm}$ right sided frontoparietal parasagittal extraaxial mass. Tumor showed intermedier signals in T2, low signal intensities in T1 and low contrast enhancing (Figure 1-2). Cranial venous MR angiography was normal.

The patient underwent a major surgery with parietal craniotomy. The dura mater was tense. After opening the dura mater, white coloured, harsh tumor was seen. Since the tumor originated from the falx, it was designated as an extra-axial mass. But the colour and consistency was different from a typical meningioma according to surgeons peroperative impression. The tumor located in right frontoparietal area was totally removed. Macroscopic examination revealed $6 \times 5 \times 2 \mathrm{~cm}$ fragmanted, focally hemorrhagic white coloured material. All the material was processed and examined.

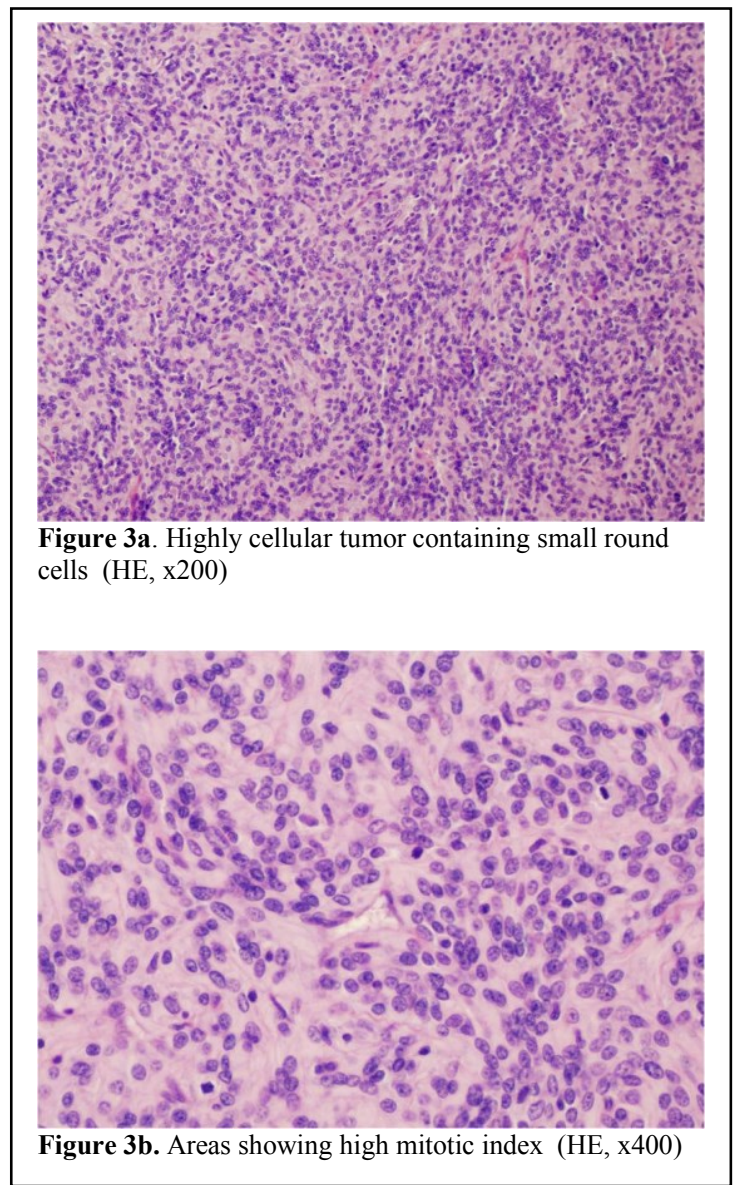

The tumoral excision material did not contain normal brain parenchyma. Histopathologic examination showed highly cellular lesion. Monotonous, oval-round small cells were forming whorl structures (Figure 3a). There was no marked atypia and necrosis but high mitotic index was evident (Figure 3b).

Most of the areas showed 10-15 mitosis/10 high power field (HPF) but focal areas showed 22 mitosis/10 HPF. Ki 67 proliferation index was 20$30 \%$. Immunohistochemical analysis showed EMA, Vimentin, Progesterone receptor (PR), bcl-2 positivity; GFAP, chromogranin, synaptophysin, S100, CD34 negativity. Our final final diagnosis according to histopathology, immunohistochemistry and radiology was anaplastic meningioma (WHO Grade 3). Postoperative follow-up computed tomography scans showed no residual tumor. The patient had no remnant neurological deficit after operation

\section{Discussion}

Childhood meningiomas show more aggressive biological potential than adult counterparts. They tend to grow more rapidly and are likely to recur in a short period. The size of tumors is also larger in children [1, 11]. Anaplastic or malignant meningiomas are classified as WHO grade III and 
account for $1 \%-3 \%$ in adults while it reaches to $4.7-7.2 \%$ in children [1].

These tumors show frank anaplastic features or high mitotic index ( $\geq 20$ mitoses/10 HPF). Grade III tumors have higher frequencies of local invasion, recurrence, and metastasis. Prognosis is poor in this group of patients, with recurrence rates of $50 \%-80 \%$ and a median survival time $<2$ years. The extent of resection is the strongest independent prognostic factor for children [9].

Recurrence free survival rates (RFS) improve significantly with gross total excision [9]. Here we present an anaplastic meningioma case in whom the diagnosis relied on high mitotic activity. Neuroradiological findings also differ from adult meningiomas. Inhomogeneous enhancement of the tumor in $36.2 \%$ of the patients might lead to misdiagnosis for glioma [7]. Though the localization was typical, low contrast enhancing was an atypical feature in our case.

Progesterone receptor positivity is inversely correlated with histologic grade and mitotic index in meningiomas. In univariate and multivariate analysis, a negative progesterone receptor combined with strong Bcl-2 immunoreactivity were significantly related to RFS [12]. Our case showed strong intensity with PR antibody. This IHC profile is rare for high grade meningiomas. Long term follow up will show the impact of high, intense PR positivity in our case.

We report this case as literature data on high grade meningiomas is limited to small case series and individual cases. Validated guidelines of management strategies might be established through these reports including long-term follow up of the cases

Financial Support: This research received no specific grant from any funding agency, commercial or not-for-profit sectors

Conflict of Interest: The authors declare no potential conflicts of interest with respect to the research, authorship, and/or publication of this article.

\section{References}

1. Kotecha RS, Junckerstorff RC, Lee S, Cole CH, Gottardo NG. Pediatric meningioma: current approaches and future direction. Journal of neuro-oncology. 2011;104(1):1-10.

2. Mekitarian Filho E, Horigoshi NK, Carvalho WB, Hirscheimer MR, Bresolin AU, Leme RJ, et al. Primary spinal meningioma in a 10-year-old boy. Arquivos de neuro-psiquiatria. 2010;68(5):804-6.

3. Riemenschneider MJ, Perry A, Reifenberger G. Histological classification and molecular genetics of meningiomas. The Lancet Neurology. 2006;5(12):104554.

4. Liu Y, Li F, Zhu S, Liu M, Wu C. Clinical features and treatment of meningiomas in children: report of 12 cases and literature review. Pediatric neurosurgery. 2008:44(2):112-7.

5. Menon G, Nair S, Sudhir J, Rao BR, Mathew A, Bahuleyan B. Childhood and adolescent meningiomas: a report of 38 cases and review of literature. Acta neurochirurgica. 2009;151(3):239-44; discussion 44.

6. Arivazhagan A, Devi BI, Kolluri SV, Abraham RG, Sampath S, Chandramouli BA. Pediatric intracranial meningiomas--do they differ from their counterparts in adults? Pediatric neurosurgery. 2008;44(1):43-8.

7. Gao X, Zhang R, Mao Y, Wang Y. Childhood and juvenile meningiomas. Child's nervous system : ChNS : official journal of the International Society for Pediatric Neurosurgery. 2009;25(12):1571-80.

8. Greene S, Nair N, Ojemann JG, Ellenbogen RG, Avellino AM. Meningiomas in children. Pediatric neurosurgery. 2008;44(1):9-13

9. Kotecha RS, Pascoe EM, Rushing EJ, Rorke-Adams LB, Zwerdling T, Gao X, et al. Meningiomas in children and adolescents: a meta-analysis of individual patient data. The Lancet Oncology. 2011;12(13):1229-39.

10. Kohama I, Sohma T, Nunomura K, Igarashi K, Ishikawa A. Intraparenchymal meningioma in an infant--case report. Neurologia medico-chirurgica. 1996;36(8):598601

11. Santos MV, Furlanetti L, Valera ET, Brassesco MS, Tone LG, de Oliveira RS. Pediatric meningiomas: a singlecenter experience with 15 consecutive cases and review of the literature. Child's nervous system : ChNS : official journal of the International Society for Pediatric Neurosurgery. 2012;28(11):1887-96

12. Wang XQ, Jiang CC, Zhao L, Gong Y, Hu J, Chen H. Erratum: Clinical features and treatment of World Health Organization Grade II and III meningiomas in childhood: report of 23 cases. Journal of neurosurgery Pediatrics. 2012;10(5):459.

Copyright (C) 2014 The Author(s); This is an open-access article distributed under the terms of the Creative Commons Attribution License (http://creativecommons.org/licenses/by/4.0), which permits unrestricted use, distribution, and reproduction in any medium, provided the original work is properly cited. 